\title{
Central Notations for the Revised ISCC-NBS Color-Name Blocks
}

\author{
Kenneth L. Kelly
}

\begin{abstract}
Nickerson and Newhall published, in 1941, the central notations of the original ISCCNBS (Inter-Society Color Council-National Bureau of Standards) color-name blocks which were used in the preparation of the soil and rock color-name charts. In 1955, the ISCCNBS color-name blocks were revised to accord more closely with usage in the textile and other fields (NBS Circular 553). The central notations of these revised color-name blocks have been computed and are given in the present paper in tabular form. A color chart showing the central colors of the 267 ISCC-NBS color-name blocks would serve for rapid determination of the ISCC-NBS color designation, especially in field work where speed and ease of operation are more important than high accuracy.
\end{abstract}

\section{Introduction}

In 1941 Nickerson and Newhall published a paper []$^{1}$ entitled Central Notations for ISCC-NBS Color Names based on the first description of the ISCC-NBS method of describing colors published in 1939 [2]. Since then, the ISCC-NBS color-name charts have been revised to accord more closely with usage in the textile and other fields and have been published in 1955 under the title The ISCC-NBS Method of Designating Colors and a Dictionary of Color Names [3]. The present paper contains the central notations of the revised color-name blocks.

\section{Computation of Centroids}

The color-name blocks into which the color solid has been divided are bounded at top and bottom by planes of constant Munsell value, at two of the sides by planes of constant Munsell hue, and at the other two sides by concentric cylindrical surfaces of constant Munsell chroma. The shape of such a block might be called a sector of a right cylindrical annulus (like a piece of pie with the point bitten off). The simplest such block is thus defined by six limits: two-value, two-hue, and two-chroma limits; the most complicated of these blocks may be broken down into three blocks of this simplest or elementary shape. The general method is to find the centroid of each such block and then find the volume-weighted centroid of the set of one to three such elementary centroids.

The hue and value of such an elementary centroid are simply the averages of the hue and value limits, respectively. The chroma of the centroid is not entirely a function of the chroma limits, but depends on the number of Munsell-hue steps $\Delta H$ in the block. Let the cross section of the block at constant Munsell value be laid out in cylindrical coordinates with the radius being Munsell chroma, $C$, and since there are 100 Munsell-hue steps in the complete hue circuit, the angle $\Delta \theta$ in radians will be given in terms of Munsell hue, $H$, as $2 \pi \Delta H / 100$. Further let $H=0$ correspond to the Munsell hue, $\bar{H}$, of the centroid of the block.

\footnotetext{
1 Figures in brackets indicate the literature references at the end of this paper.
}

The chroma, $\bar{C}$, of the centroid [1] is defined as:

$$
\begin{gathered}
\bar{C} \equiv \frac{\int_{\frac{\theta}{2}}^{\frac{\theta}{2}} \int_{C_{n}}^{C_{x}} C^{2}(\cos \theta) d C d \theta}{\int_{-\frac{\theta}{2}}^{\frac{\theta}{2}} \int_{C_{n}}^{C_{x}} C d C d^{2} \theta}, \text { or } \\
\bar{C}=\frac{2\left(C_{x}^{2}+C_{x}^{y} C_{n}+C_{n}^{2}\right) \sin (\pi \Delta H / 100)}{3\left(C_{x}+C_{n}\right)(\pi \Delta H / 100)},
\end{gathered}
$$

where $C_{x}$ and $C_{n}$ are the upper and lower chroma limits of the block, respectively.

By this method, the central notation $(\bar{H}, \bar{V} / \bar{C})$ of an elementary block like moderate red, hue range $1 R$ to $7 R$, value range from 3.5 to 5.5 , and chroma range from 7 to 11 , is determined as:

$$
\bar{H}=(1 R+7 R) / 2=4 R ; \bar{V}=(3.5+5.5) / 2=4.5,
$$

and

$$
\begin{aligned}
\bar{C}=\frac{2\left(11^{2}+7 \times 11+7^{2}\right) \sin (6 \pi / 100)}{3(11+7)(6 \pi / 100)} & \\
& =\frac{2 \times 247 \times 0.1874}{3 \times 18 \times 0.1885}=9.1
\end{aligned}
$$

The central notation of a compound block, that is, one in which two or more elementary blocks are joined together along common constant-hue planes, such as yellowish white or moderate blue, is found by computing the volume-weighted mean of the central notations of the simple blocks. The centroid of a two-component block, for instance, will lie on the straight line joining the centroids of the component blocks. Therefore, it is necessary to graph the respective centroids on circular graph paper, connect them by a straight line divided inversely according to the two volumes, and read off the hue and chroma of the resultant centroid. For a threecomponent block, the resultant centroid for two of the adjacent blocks is found and then combined by the same method with the centroid of the third block. The value notation of the resultant centroid is computed as the volume-weighted mean of the value notations of the separate centroids. 


\section{Peripheral Color-Name Blocks}

The shape of the psychological color solid may be described as a grossly misshapen grapefruit, set so that the pithy core is vertical with an irregular outer surface or skin, determined by the MacAdam theoretical pigment limits [4]. The bounding surface has been determined in Munsell renotation terms by Nickerson and Newhall [8]. This solid, cut into 267 blocks each with a color name attached, forms the ISCC-NBS system of color names. Now consider the outermost or peripheral blocks, those that extend to the outer surface or skin. These represent colors of maximum saturation, maximum lightness or minimum lightness as determined from the MacAdam limits. These peripheral blocks, of which there are 120, have several simple boundary surfaces (plane and cylindrical) and are closed by the outer complicated curved surface of the color solid. The centroids of these peripheral blocks have been estimated graphically by plotting the MacAdam limits on the color-name charts.

\section{Table of Central Notations of Color- Name Blocks}

In table 1 the ISCC-NBS color-name blocks are identified by number and name, and the Munsell renotation of each centroid is given. The central notation of each of the 120 peripheral blocks, less certain because it was estimated rather than computed by formula, is marked with an asterisk. The numbers used to identify and order the color designations in table 1 are taken from table 1 of NBS Circular 553 [3]. Thus, for any hue name, the order of modifiers is vivid; brilliant, strong, deep, very deep; very light, light, moderate, dark, very dark; very pale (very light grayish), pale (light grayish), grayish, dark grayish, blackish; -ish white, light -ish gray; -ish gray, dark -ish gray, and -ish black. Not all of these modifiers are required with every hue name, as for instance there is no very dark pink or very pale olive. The notations of the centroids are given to one decimal place except that the value or chroma notations of the centroids of some of the peripheral blocks are given as whole numbers followed by a plus or minus sign, such as $4.5 Y R 6.8 / 16+$, indicating uncertainty in the next decimal place.

TABLE 1. Central notations of ISCC-NBS color-name blocks.

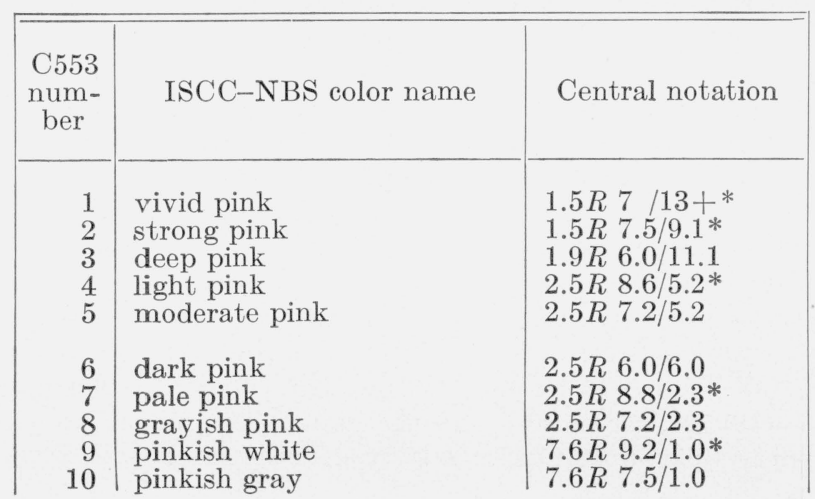

TABLE 1. Central notations of ISCC-NBS color-name blocks.-Continued

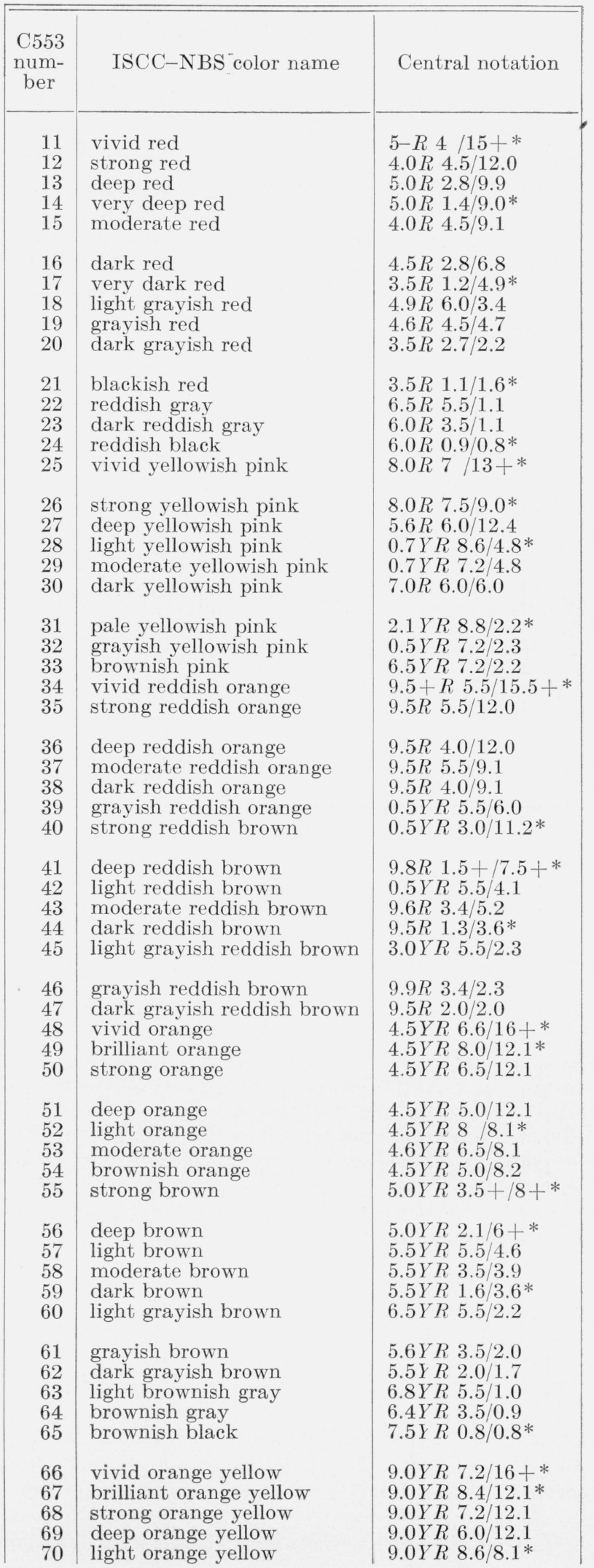


TABLE 1. Central notations of ISCC-NBS color-name blocks.-Continued

\begin{tabular}{|c|c|c|}
\hline $\begin{array}{l}\text { C553 } \\
\text { num- } \\
\text { ber }\end{array}$ & ISCC-NBS color name & Central notation \\
\hline $\begin{array}{l}71 \\
72 \\
73 \\
74 \\
75\end{array}$ & $\begin{array}{l}\text { moderate orange yellow } \\
\text { dark orange yellow } \\
\text { pale orange yellow } \\
\text { strong yellowish brown } \\
\text { deep yellowish brown }\end{array}$ & $\begin{array}{l}9.0 Y R \quad 7.2 / 8.1 \\
9.0 Y R 6.0 / 8.1 \\
9.1 Y R \quad 8.6 / 4.4^{*} \\
9.0 Y R \quad 4.6 / 9.6^{*} \\
9.5 Y R 2.9 / 6+*\end{array}$ \\
\hline $\begin{array}{l}76 \\
77 \\
78 \\
79\end{array}$ & $\begin{array}{l}\text { light yellowish brown } \\
\text { moderate yellowish brown } \\
\text { dark yellowish brown } \\
\text { light grayish yellowish } \\
\text { brown } \\
\text { grayish yellowish brown }\end{array}$ & $\begin{array}{l}9.2 Y R \quad 6.6 / 4.7 \\
9.5 Y R 4.5 / 4.0 \\
9.5 Y R 2.2 / 3.7 * \\
9.5 Y R 6.4 / 2.4 \\
9.5 Y R \quad 4.6 / 2.1\end{array}$ \\
\hline $\begin{array}{l}81 \\
82 \\
83 \\
84 \\
85\end{array}$ & $\begin{array}{l}\text { dark grayish yellow brown } \\
\text { vivid yellow } \\
\text { brilliant yellow } \\
\text { strong yellow } \\
\text { deep yellow }\end{array}$ & $\begin{array}{l}9.5 Y R 2.4 / 1.8 \\
4.0 Y 7.8 / 14.5+* \\
4.0 Y 8.8 / 9.5 * \\
4.0 Y 7.2 / 9.5 \\
4.0 Y 6.0 / 9.5\end{array}$ \\
\hline $\begin{array}{l}86 \\
87 \\
88 \\
89 \\
90\end{array}$ & $\begin{array}{l}\text { light yellow } \\
\text { moderate yellow } \\
\text { dark yellow } \\
\text { pale yellow } \\
\text { grayish yellow }\end{array}$ & $\begin{array}{l}4.0 Y 8.8 / 6.6 * \\
4.0 Y 7.2 / 6.6 \\
4.0 Y 6.0 / 6.6 \\
4.2 Y 8.9 / 3.6 * \\
4.2 Y 7.2 / 3.6\end{array}$ \\
\hline $\begin{array}{l}91 \\
92 \\
93 \\
94 \\
95\end{array}$ & $\begin{array}{l}\text { dark grayish yellow } \\
\text { yellowish white } \\
\text { yellowish gray } \\
\text { light olive brown } \\
\text { moderate olive brown }\end{array}$ & $\begin{array}{l}4.0 Y 6.0 / 4.1 \\
4.1 Y 9.2 / 1.3 * \\
4.1 Y 7.5 / 1.3 \\
2.5 Y 5.0 / 8.4 * \\
2.5 Y 3.5+/ 6+*\end{array}$ \\
\hline $\begin{array}{r}96 \\
97 \\
98 \\
99 \\
100\end{array}$ & $\begin{array}{l}\text { dark olive brown } \\
\text { vivid greenish yellow } \\
\text { brilliant greenish yellow } \\
\text { strong greenish yellow } \\
\text { deep greenish yellow }\end{array}$ & $\begin{array}{l}2.5 Y 1.8 / 2.5+* \\
9.5 Y 7.8 / 14.5+* \\
9.5 Y 8.8 / 9.5 * \\
9.5 Y 7.2 / 9.5 \\
9.5 Y 6.0 / 9.5\end{array}$ \\
\hline $\begin{array}{l}101 \\
102 \\
103 \\
104 \\
105\end{array}$ & $\begin{array}{l}\text { light greenish yellow } \\
\text { moderate greenish yellow } \\
\text { dark greenish yellow } \\
\text { pale greenish yellow } \\
\text { grayish greenish yellow }\end{array}$ & $\begin{array}{l}9.5 Y 8.8 / 6.6 * \\
9.5 Y 7.2 / 6.6 \\
9.5 Y 6.0 / 6.6 \\
9.5 Y 8.9 / 4.1 * \\
9.5 Y 7.2 / 4.1\end{array}$ \\
\hline $\begin{array}{l}106 \\
107 \\
108 \\
109 \\
110\end{array}$ & $\begin{array}{l}\text { light olive } \\
\text { moderate olive } \\
\text { dark olive } \\
\text { light grayish olive } \\
\text { grayish olive }\end{array}$ & $\begin{array}{l}8.1 Y 5.1 / 8.0 * \\
8.0 Y 3.5+/ 5.8+* \\
8.0 Y 1.7 / 3.2 * \\
8.3 Y 5.5 / 2.4 \\
8.1 Y 3.5 / 2.3\end{array}$ \\
\hline $\begin{array}{l}111 \\
112 \\
113 \\
114 \\
115\end{array}$ & $\begin{array}{l}\text { dark grayish olive } \\
\text { light olive gray } \\
\text { olive gray } \\
\text { olive black } \\
\text { vivid yellow green }\end{array}$ & $\begin{array}{l}8.0 Y 2.0 / 2.0 \\
7.4 Y 5.5 / 1.3 \\
8.1 Y 3.5 / 1.0 \\
9.0 Y 0.9 / 0.8 * \\
5.0 G Y 7.5 / 14.5+*\end{array}$ \\
\hline $\begin{array}{l}116 \\
117 \\
118 \\
119 \\
120\end{array}$ & $\begin{array}{l}\text { brilliant yellow green } \\
\text { strong yellow green } \\
\text { deep yellow green } \\
\text { light yellow green } \\
\text { moderate yellow green }\end{array}$ & $\begin{array}{l}5.0 G Y 8.5 / 9.1 * \\
5.0 G Y 6.0 / 9.1 \\
5.0 G Y 4.0 / 9.1 \\
5.0 G Y 8.6 / 5.2^{*} \\
5.0 G Y 6.0 / 5.2\end{array}$ \\
\hline $\begin{array}{l}121 \\
122 \\
123 \\
124 \\
125\end{array}$ & $\begin{array}{l}\text { pale yellow green } \\
\text { grayish yellow green } \\
\text { strong olive green } \\
\text { deep olive green } \\
\text { moderate olive green }\end{array}$ & $\begin{array}{l}3.5 G Y 8.7 / 2.2 * \\
4.4 G Y 6.1 / 2.2 \\
5.0 G Y 3.2 / 7.8+* \\
5.0 G Y 2.4+/ 7.1+* \\
5.0 G Y 3.5 / 5.2\end{array}$ \\
\hline $\begin{array}{l}126 \\
127 \\
128 \\
129 \\
130\end{array}$ & $\begin{array}{l}\text { dark olive green } \\
\text { grayish olive green } \\
\text { dark grayish olive green } \\
\text { vivid yellowish green } \\
\text { brilliant yellowish green }\end{array}$ & $\begin{array}{l}5.0 G Y \quad 1.8 / 3.7 * \\
5.0 G Y 3.5 / 2.2 \\
5.0 G Y 2.0 / 2.0 \\
0.5 G 7 / 15.5+* \\
0.5 G 8.0 / 9.1 *\end{array}$ \\
\hline
\end{tabular}

TABLE 1. Centrat notations of ISCC-NBC color-name blocks.-Continued

\begin{tabular}{|c|c|c|}
\hline $\begin{array}{c}\text { C553 } \\
\text { num- } \\
\text { ber }\end{array}$ & ISCC-NBS color name & Central notation \\
\hline $\begin{array}{l}131 \\
132 \\
133 \\
134 \\
135\end{array}$ & $\begin{array}{l}\text { strong yellowish green } \\
\text { deep yellowish green } \\
\text { very deep yellowish green } \\
\text { very light yellowish green } \\
\text { light yellowish green }\end{array}$ & $\begin{array}{l}0.5 G \text { S } 5.5 / 9.1 \\
0.5 G 3.5 / 11.5+* \\
0.5 G 2.0 / 9.2+* \\
0.5 G 9.1 / 5.1 * \\
0.5 G \quad 7.5 / 5.1\end{array}$ \\
\hline $\begin{array}{l}136 \\
137 \\
138 \\
139 \\
140\end{array}$ & $\begin{array}{l}\text { moderate yellowish green } \\
\text { dark yellowish green } \\
\text { very dark yellowish green } \\
\text { vivid green } \\
\text { brilliant green }\end{array}$ & $\begin{array}{l}0.5 G 5.5 / 5.1 \\
0.5 G 3.5 / 5.1 \\
0.5 G 1.5 / 4.9 * \\
6.5 G 5.2 / 18+* \\
6.0 G 7.4 / 9.1 *\end{array}$ \\
\hline $\begin{array}{l}141 \\
142 \\
143 \\
144 \\
145\end{array}$ & $\begin{array}{l}\text { strong green } \\
\text { deep green } \\
\text { very light green } \\
\text { light green } \\
\text { moderate green }\end{array}$ & $\begin{array}{l}6.0 G 4.5 / 9.1 \\
6.0 G 2.3 / 9.1 * \\
6.0 G 8.6 / 5.1 * \\
6.0 G 6.5 / 5.1 \\
6.0 G 4.5 / 5.1\end{array}$ \\
\hline $\begin{array}{l}146 \\
147 \\
148 \\
149 \\
150\end{array}$ & $\begin{array}{l}\text { dark green } \\
\text { very dark green } \\
\text { very pale green } \\
\text { pale green } \\
\text { grayish green }\end{array}$ & $\begin{array}{l}6.0 G 2.7 / 5.0 \\
6.0 G 1.3 / 4.9 * \\
9.0 G 8.7 / 1.8 * \\
9.0 G 6.5 / 1.8 \\
9.0 G 4.5 / 1.8\end{array}$ \\
\hline $\begin{array}{l}151 \\
152 \\
153 \\
154 \\
155\end{array}$ & $\begin{array}{l}\text { dark grayish green } \\
\text { blackish green } \\
\text { greenish white } \\
\text { light greenish gray } \\
\text { greenish gray }\end{array}$ & $\begin{array}{l}9 . C G 2.8 / 1.6 \\
9 .(G) 1.1 / 1.4 * \\
7 .(G) 9.2 / 0.8 * \\
7 . C G 7.5 / 0.8 \\
7.0 G 5.5 / 0.8\end{array}$ \\
\hline $\begin{array}{l}156 \\
157 \\
158 \\
159 \\
160\end{array}$ & $\begin{array}{l}\text { dark greenish gray } \\
\text { greenish black } \\
\text { vivid bluish green } \\
\text { brilliant bluish green } \\
\text { strong bluish green }\end{array}$ & $\begin{array}{l}7.0 G 3.5 / 0.8 \\
7.5 G 0.9 / 0.7 * \\
4.5 B G 5.3 / 15.5+* \\
4.5 B G 7.3 / 9.0 * \\
4.5 B G 4.5 / 9.0\end{array}$ \\
\hline $\begin{array}{l}161 \\
162 \\
163 \\
164 \\
165\end{array}$ & $\begin{array}{l}\text { deep bluish green } \\
\text { very light bluish green } \\
\text { light bluish green } \\
\text { moderate bluish green } \\
\text { dark bluish green }\end{array}$ & $\begin{array}{ll}4.5 B G & 2.3 / 9.0^{*} \\
4.5 B G & 8.5 / 5.0^{*} \\
4.5 B G & 6.5 / 5.0 \\
4.5 B G & 4.5 / 5.0 \\
4.5 B G & 2.7 / 5.0\end{array}$ \\
\hline $\begin{array}{l}166 \\
167 \\
168 \\
169 \\
170\end{array}$ & $\begin{array}{l}\text { very dark bluish green } \\
\text { vivid greenish blue } \\
\text { brilliant greenish blue } \\
\text { strong greenish blue } \\
\text { deep greenish blue }\end{array}$ & 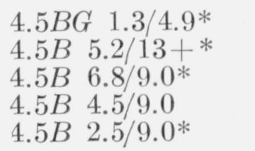 \\
\hline $\begin{array}{l}171 \\
172 \\
173 \\
174 \\
175\end{array}$ & $\begin{array}{l}\text { very light greenish blue } \\
\text { light greenish blue } \\
\text { moderate greenish blue } \\
\text { dark greenish blue } \\
\text { very dark greenish blue }\end{array}$ & $\begin{array}{ll}4.5 B & 8.2 / 5.2 * \\
4.5 B & 6.5 / 5.2 \\
4.5 B & 4.5 / 5.2 \\
4.5 B & 2.7 / 5.0 \\
4.5 B & 1.3 / 4.9 *\end{array}$ \\
\hline $\begin{array}{l}176 \\
177 \\
178 \\
179 \\
180\end{array}$ & $\begin{array}{l}\text { vivid blue } \\
\text { brilliant blue } \\
\text { strong blue } \\
\text { deep blue } \\
\text { very light blue }\end{array}$ & $\begin{array}{ll}3.0 P B & 4.2 / 15+* \\
3.0 P B & 6.4 / 11.0 * \\
3.0 P B & 4.2 / 11.0 \\
3.0 P B & 2.0 / 9.0 * \\
2.4 P B & 8.2 / 7.2 *\end{array}$ \\
\hline $\begin{array}{l}181 \\
182 \\
183 \\
184 \\
185\end{array}$ & $\begin{array}{l}\text { light blue } \\
\text { moderate blue } \\
\text { dark blue } \\
\text { very pale blue } \\
\text { pale blue }\end{array}$ & $\begin{array}{ll}2.7 P B & 6.5 / 7.3 \\
2.9 P B & 4.2 / 7.2 \\
2.8 P B & 1.7 / 5.0^{*} \\
0.9 P B & 8.4 / 3.0^{*} \\
0.9 P B & 6.5 / 3.0\end{array}$ \\
\hline $\begin{array}{l}186 \\
187 \\
188 \\
189 \\
190\end{array}$ & $\begin{array}{l}\text { grayish blue } \\
\text { dark grayish blue } \\
\text { blackish blue } \\
\text { bluish white } \\
\text { light bluish gray }\end{array}$ & 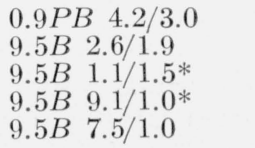 \\
\hline
\end{tabular}


TABLE 1. Central notations of ISCC-NBS color-name blocks - Continued

\begin{tabular}{|c|c|c|}
\hline $\begin{array}{l}\text { C553 } \\
\text { num- } \\
\text { ber }\end{array}$ & ISCC-NBS color name & Central notation \\
\hline 191 & bluish gray & $9.5 B \quad 5.5 / 1.0$ \\
\hline 192 & dark bluish gray & $9.5 B \quad 3.5 / 1.0$ \\
\hline 193 & bluish black & $9.5 B \quad 1.0 / 0.7 *$ \\
\hline 194 & vivid purplish blue & $8.0 P B 2.5 / 22+*$ \\
\hline 195 & brilliant purplish blue & $8.0 P B \quad 6.0 / 10.8 *$ \\
\hline 196 & strong purplish blue & $8.0 P B \quad 4.1 / 11.4$ \\
\hline 197 & deep purplish blue & $8.0 P B \quad 1.6 / 9.1 *$ \\
\hline 198 & very light purplish blue & 7. $P B 7.8+/ 6.1+*$ \\
\hline 199 & light purplish blue & $7.5 P B \quad 6.0 / 6.8$ \\
\hline 200 & moderate purplish blue & $8.0 P B \quad 3.5 / 6.9$ \\
\hline 201 & dark purplish blue & $7.9 P B \quad 1.1 / 4.7^{*}$ \\
\hline 202 & very pale purplish blue & $7.0 P B 8.2 / 4.1 *$ \\
\hline 203 & pale purplish blue & $7.0 P B \quad 6.0 / 4.1$ \\
\hline 204 & grayish purplish blue & $\begin{array}{lll}7.2 P B & 3.3 / 4.0\end{array}$ \\
\hline 205 & vivid violet & $1.0 P 2.7 / 21+*$ \\
\hline 206 & brilliant violet & $1.0 P 5.9 / 11.1 *$ \\
\hline 207 & strong violet & $1.0 P \quad 3.5 / 11.1$ \\
\hline 208 & deep violet & $1.0 P 1.4 / 10.3^{*}$ \\
\hline 209 & very light violet & $1.0 P 7.9 / 7 *$ \\
\hline 210 & light violet & $1.0 P \quad 6.0 / 7.2$ \\
\hline 211 & moderate violet & $1.0 P \quad 3.5 / 7.2$ \\
\hline 212 & dark violet & $1.0 P 1.3 / 5.0 *$ \\
\hline 213 & very pale violet & $1.0 P 8.2 / 4.1 *$ \\
\hline 214 & pale violet & $1.0 P 6.0 / 4.1$ \\
\hline 215 & grayish violet & $1.0 P \quad 3.3 / 4.1$ \\
\hline 216 & vivid purple & $6.0 P 3.5 / 20+*$ \\
\hline 217 & brilliant purple & $6.0 P 6.7 / 11.1 *$ \\
\hline 218 & strong purple & $6.0 P \quad 4.5 / 11.1$ \\
\hline 219 & deep purple & $6.0 P \quad 2.8 / 10.2$ \\
\hline 220 & very deep purple & $6.0 P \quad 1.2 / 10.2 *$ \\
\hline 221 & very light purple & $6.0 P 8.0 / 7.2 *$ \\
\hline 222 & light purple & $6.0 P \quad 6.5 / 7 / 2$ \\
\hline 223 & moderate purple & $6.0 P \quad 4.5 / 7.2$ \\
\hline 224 & dark purple & $6.0 P \quad 2.7 / 5.1$ \\
\hline 225 & very dark purple & $6.0 P \quad 1.1 / 4.9 *$ \\
\hline 226 & very pale purple & $5.4 P$ 8.4/3.3* \\
\hline 227 & pale purple & $6.8 P \quad 6.4 / 3.0$ \\
\hline 228 & grayish purple & $7.8 P \quad 4.5 / 2.9$ \\
\hline 229 & dark grayish purple & $10.0 P \quad 2.8 / 2.0$ \\
\hline 230 & blackish purple & $10.0 P 1.0 / 1.4 *$ \\
\hline
\end{tabular}

\section{Applications}

For nonborderline colors it is much easier to determine the ISCC-NBS color name from a color chart containing 267 samples, one for each of the central notations of the 267 ISCC-NBS color-name blocks, than it is to determine the Munsell renotation of the color and then to find the ISCC-NBS designation from the color-name charts. In using such a color chart, the observer simply picks out the particular color most closely duplicating that of the specimen to be designated; he does not have to check for conformity to hue, lightness, and saturation limits separately. The production of such a color chart is now under consideration. It should be pointed out, however, that if the specimen color falls about equally near to two or more of the 267 central colors, it would be impossible to determine
TABLE 1. Central notations of ISCC-NBS color-name blocks.-Continued

\begin{tabular}{|c|c|c|}
\hline $\begin{array}{c}\text { C553 } \\
\text { num- } \\
\text { ber }\end{array}$ & ISCC-NBS color name & Central notations \\
\hline 231 & purplish white & $9.0 P 9.1 / 1.0 *$ \\
\hline 232 & light purplish gray & $9.0 P \quad 7.5 / 1.0$ \\
\hline 233 & purplish gray & $10.0 P \quad 5.5 / 1.0$ \\
\hline 234 & dark purplish gray & $10.0 P \quad 3.5 / 1.0$ \\
\hline 235 & purplish black & $10.0 P 1.0 / 0.7 *$ \\
\hline 236 & vivid reddish purple & $1.0 R P 4+/ 19+*$ \\
\hline 237 & strong reddish purple & $1.0 R P \quad 4.5 / 11.1$ \\
\hline 238 & deep reddish purple & $1.0 R P 2.8 / 10.3^{\prime}$ \\
\hline 239 & very deep reddish purple & $1.0 R P 1.3 / 10.3 *$ \\
\hline 240 & light reddish purple & $1.0 R P \quad 6.0 / 7.2$ \\
\hline 241 & moderate reddish purple & $1.0 R P \quad 4.5 / 7.2$ \\
\hline 242 & dark reddish purple & $1.0 R P \quad 2.7 / 5.1$ \\
\hline 243 & very dark reddish purple & $1.0 R P 1.1 / 5.0 *$ \\
\hline 244 & pale reddish purple & $1.0 R P \quad 6.0 / 4.1$ \\
\hline 245 & grayish reddish purple & $1.0 R P \quad 4.5 / 4.1$ \\
\hline 246 & brilliant purplish pink & $4.0 R P \quad 7.9+/ 11+*$ \\
\hline 247 & strong purplish pink & $4.0 R P 7-/ 14.5^{*}$ \\
\hline 248 & deep purplish pink & $4.0 R P \quad 6.0 / 12.0$ \\
\hline 249 & light purplish pink & $4.0 R P \quad 8.3 / 7.1 *$ \\
\hline 250 & moderate purplish pink & $4.0 R P \quad 7.0 / 7.1$ \\
\hline 251 & dark purplish pink & $6.0 R P \quad 6.0 / 7.2$ \\
\hline 252 & pale purplish pink & $4.0 R P 8.5 / 3.5^{*}$ \\
\hline 253 & grayish purplish pink & $4.0 R P \quad 7.0 / 3.5$ \\
\hline 254 & vivid purplish red & $7.0 R P 4+/ 17+*$ \\
\hline 255 & strong purplish red & $7.0 R P \quad 4.5 / 11.9$ \\
\hline 256 & deep purplish red & $7.0 R P \quad 2.8 / 11.0$ \\
\hline 257 & very deep purplish red & 7.0RP $1.3 / 9.0 *$ \\
\hline 258 & moderate purplish red & $7.0 R P 4.5 / 9.0$ \\
\hline 259 & dark purplish red & $7.0 R P \quad 2.7 / 6.3$ \\
\hline 260 & very dark purplish red & $7.0 R P 1.2 / 4.9 *$ \\
\hline 261 & light grayish purplish red & $7.0 R P \quad 6.0 / 4.0$ \\
\hline 262 & grayish purplish red & $7.0 R P \quad 4.5 / 5.2$ \\
\hline 263 & white & $3.0 Y 9.25 / 0.06$ \\
\hline 264 & light gray & $3.0 Y 7.5 / 0.06$ \\
\hline 265 & medium gray & $3.0 Y \quad 5.5 / 0.06$ \\
\hline 266 & dark gray & $N 3.5 /$ \\
\hline 267 & black & $N 1.25 /$ \\
\hline
\end{tabular}

accurately which ISCC-NBS designation to apply to it. If necessary, such borderline cases would have to be resolved by recourse to the method described in NBS Circular 553 [3].

These central notations have a number of applications. Those published by Nickerson and Newhall [7] were used in designing the color-name charts used in the description of the colors of soils [5] and rocks [6]. In both of these color charts, the Munsell Color Company painted samples representing the central notations of each of the color-name blocks used. Matching to one central color rather than to several limit colors is a less exact but quicker method and was developed for field use where speed and ease of operation are more important than high accuracy.

The author acknowledges the assistance received from G. L. Gibson in checking the computation of the centroid locations. 


\section{References}

[1] Dull, R. W., Mathematics for engineers, p. 753 (McGrawHill Book Company Ine., N. Y., N. Y., 1926).

[2] Judd, D. B., and Kelly, K. L., Method of designating colors, J. Research NBS 23, 355 (1939) RP1239.

[3] Kelly, K. L. and Judd, D. B., The ISCC-NBS method of designating colors and a dictionary of color names, NBS Circ, 553 (1955), obtainable from the Superintendent of Documents, U. S. Government Printing Office, Washington 25 , D. C. $(\$ 2.00)$.

[4] MacAdam, D. L., Maximum visual efficiency of colored materials, J. Opt. Soc. Am. 25, 361 (1935).

[5] Munsell Color Company, Munsell Soil Color Charts, special form for use of soil scientists, geologists, and archaeologists (Munsell Color Company, 10 East Franklin Street, Baltimore 2, Md., 1950).
[6] National Research Council, Rock-Color Chart, prepared by the Rock-Color Chart Committee (sponsored by five geological organizations), National Research Council, Washington, D. C., 1948.

[7] Nickerson, Dorothy, and Newhall, Sidney M., Central notations for ISCC-NBS color names, J. Opt. Soc. Am. 31, 587 (1941).

[8] Nickerson, Dorothy, and Newhall, Sidney M., A psychological color solid, J. Opt. Soc. Am. 33, 419 (1943).

Washington, June 3, 1958. 